\title{
Antimicrobial stewardship in Victorian hospitals: a statewide survey to identify current gaps
}

Rodney S James BMedSci, BMed, FRCPA Research Fellow and Clinical Microbiologist

Kylie A McIntosh BPharm(Hons), PhD, Quality Use of Medicines Program Manager

Susan B Luu BPharm, MPH, Research Fellow

Menino 0 Cotta BPharm,

NHMRC Research Fellow

Caroline Marshal MB BS, FRACP, PhD Clinical Research Fellow

Karin A Thursky MB BS, FRACP, MD, Infectious Disease Physician $^{3}$

Kirsty L Buising MB BS, FRACP, MD, Infectious Diseases Physician $^{3}$

1Department of Medicine University of Melbourne,

Melbourne, VIC.

2 Victorian Department of Health

Melbourne, VIC.

3 Victorian Infectious Diseases Service, Royal Melbourne Hospita Melbourne, VIC

Rod.James@mh.org.au

MJA 2013; 199: 692-695 doi: 10.5694/mjal3.10422

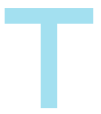

he association between antimicrobial resistance and antimicrobial use is long established. ${ }^{1-3}$ Recognition of widespread inappropriate prescription of antimicrobials in hospitals has prompted action addressing this important patient safety issue. Antimicrobial stewardship (AMS) aims to improve the quality of care and clinical outcomes of patients requiring treatment or prevention of infection, while reducing adverse events and preventing the emergence of antibiotic resistance in local pathogens. ${ }^{4}$ In 2011, the Australian Commission on Safety and Quality in Health Care (ACSQHC) published recommendations for effective AMS programs in Australian hospitals, outlining five essential AMS strategies and four additional activities (Box 1)..$^{5}$ The new National Safety and Quality Health Service (NSQHS) Standards, ${ }^{6}$ which include specific criteria for AMS, have further heightened the sense of urgency around implementing these programs.

AMS typically uses combinations of strategies, including implementing guidelines, using formularies, restriction policies, audits, education, and encouraging de-escalation of therapy and parenteral-to-oral conversion where appropriate. ${ }^{7,8}$ Successful AMS requires multidisciplinary and interdepartmental collaboration. ${ }^{9,10}$ A 2008 snapshot survey of AMS activities in Australian hospitals ${ }^{11}$ found that there was a variety of AMS programs implemented with varying degrees of success. This was a modest survey with only 80 respondents, mainly from metropolitan public hospitals, but it provided generalised insight into some programs. The aim of the current study was to perform a more in-depth survey, to describe AMS activities currently being undertaken by Victorian hospitals, and to elucidate specific gaps when assessed against the ACSQHC criteria.

\begin{abstract}
Abstrac
Objective: To determine antimicrobial stewardship (AMS) activities currently being undertaken at Victorian hospitals, identifying gaps when assessed against the Australian Commission on Safety and Quality in Health Care criteria for effective AMS.
\end{abstract}

Design, setting and participants: A survey open to all Victorian health services, conducted between January and March 2012

Main outcome measures: Availability of the endorsed prescribing guidelines, antimicrobial prescribing policies, formularies, approval systems for restricted antimicrobials, procedures for postprescription review, auditing and selective reporting of sensitivities.

Results: Response rates were $96.4 \%$ for public health services and $67.7 \%$ for private hospitals. Guidelines were available at all public and $88.1 \%$ of private hospitals, and $90.6 \%$ of public metropolitan, $45.7 \%$ of public regional and $21.4 \%$ of private hospitals had antimicrobial prescribing policies. Antimicrobial approval systems were used in $93.8 \%$ of public metropolitan, $17.3 \%$ of public regional and $4.8 \%$ of private hospitals. Prescribing audits were conducted by $62.5 \%$ of public metropolitan, $35.8 \%$ public regional and $52.4 \%$ of private hospitals. Nearly all hospitals had selective laboratory reporting of antimicrobial sensitivities. Few hospitals had dedicated funding for AMS personnel.

Conclusions: We identified wide differences between hospital AMS activities. Additional support for AMS is particularly required in the public regional and private hospital sectors, principally in the key areas of policy development, antimicrobial approval systems, prescription review and auditing. Further research is required to develop recommendations for implementation of AMS within the regional and private hospital settings.

\section{Methods}

The survey was developed by the Quality, Safety and Patient Experience Branch of the Victorian Department of Health (Vic DoH), together with the Melbourne Health AMS Research Group, consisting of infectious diseases physicians, clinical microbiologists and pharmacists. The questions were developed with reference to the ACSQHC recommendations, following a review of the literature and in conjunction with discussion among the investigators, who have extensive experience in AMS implementation. Included was information on hospital demographics, AMS activities, governance structure, resources, workforce capacity and other cultural and organisational barriers. Usability testing for the survey was conducted at six pilot hospitals, selected to ensure generalisability. Positive feedback was received regarding the ease of use and time taken to complete the survey, therefore no changes were made.

A letter was sent to the chief executives of 84 public health services and 63 private hospitals by the Vic $\mathrm{DoH}$ in November 2011, requesting a qualified staff member to respond to the survey. A link to the secure online survey, hosted by the Vic DoH, was sent via e-mail to all nominees in January 2012, and data submission closed on 31 March 2012. All Victorian public and private hospitals offering overnight stays to patients were included in the study, with mental health facilities excluded due to low levels of antimicrobial prescribing. The survey consisted of 38 questions, which were mainly close-ended, some with the option of a free-text response, and two comment-style questions at the end relating to current AMS program improvements and barriers. All questions were compulsory and completed by all respondents. As this was a self-reported 
survey, in order to improve the accuracy of the data, answers were reviewed for inconsistencies; if inconsistencies were perceived to be present, a researcher would call to seek clarification. Data were then sorted into three groups: public metropolitan, public regional and private hospitals, according to the Australian Institute of Health and Welfare remoteness classification, ${ }^{12}$ allowing the determination of possible gaps across different sectors and locations. Results were reported descriptively and no statistical analysis was performed.

As the survey was a low-risk audit and quality assurance activity, ethics approval was not required. The Vic DoH Quality, Safety and Patient Experience Branch was involved throughout the survey development, data collection and analysis. Hospital data was de-identified before reporting and involvement in the survey was voluntary.

\section{Results}

The response rate for public hospitals was $96.4 \%$ (81 of 84 eligible health service networks covering 113 sites) and $67.7 \%$ for private hospitals (42 of 62 hospitals). The overall response rate was $84.2 \%$ (123 of 146 health services). The private hospital nonrespondents did not differ systematically from respondents in geographical location, classification, co-location with a public hospital or the presence of an intensive care unit. Data on participating hospital characteristics are presented in Box 2.

Responses directly related to the ACSQHC five essential AMS strategies $^{5}$ are shown in Box 3. The consensus guidelines for prescribing in Australia, Therapeutic guidelines: antibiotic, ${ }^{13}$ were readily accessible in almost all Victorian hospitals, although endorsement of their use was lacking in hospital antimicrobial prescribing policies, particularly in public regional and private hospitals, and few hospitals had a dedicated AMS committee. Most public metropolitan hospitals had an antimicrobial formulary in place that included restrictions on broad-spectrum antimicrobials, compared with few public regional and private hospitals. Postprescription review occurred in only

\section{Effective antimicrobial stewardship ${ }^{5}$}

Five essential strategies

- Implementing clinical guidelines that are consistent with the latest version of Therapeutic guidelines: antibiotic, ${ }^{13}$ and which take into account local microbiology and antimicrobial susceptibility patterns.

- Establishing formulary restriction and approval systems that include restricting broad-spectrum and latergeneration antimicrobials to patients in whom their use is clinically justified.

- Reviewing antimicrobial prescribing with intervention and direct feedback to the prescriber - this should, at a minimum, include intensive care unit patients.

- Monitoring performance of antimicrobial prescribing by collecting and reporting unit- or ward-specific use data, auditing antimicrobial use and using quality use of medicines indicators.

- Ensuring the clinical microbiology laboratory uses selective reporting of susceptibility testing results that is consistent with hospital antimicrobial treatment guidelines.

Activities that may be undertaken depending on local priorities and available resources

- Educating prescribers, pharmacists and nurses about good antimicrobial prescribing practice and antimicrobial resistance.

- Using point-of-care interventions, including streamlining or de-escalation of therapy, dose optimisation or parenteral-to-oral conversion.

- Using information technology such as electronic prescribing with clinical decision support or online approval systems.

- Annually publishing facility-specific antimicrobial susceptibility data.

half the hospitals surveyed. Only $5.2 \%$ of hospitals had a dedicated antimicrobial management team (a multidisciplinary team involving at least one doctor and one pharmacist or nurse) in place. Performance monitoring through regular antimicrobial audits and providing feedback to prescribers was carried out by less than half the hospitals surveyed. A very high proportion of hospitals reported receiving selective antimicrobial susceptibility results from the microbiology laboratory.

Responses to supplementary AMS strategies recommended by the ACSQHC $^{5}$ and the governance structure for AMS programs are presented in Box 4. Data on point-ofcare interventions were not included in the analysis, as these questions were answered poorly, due to ambiguity with interpreting the word "regularly". Most public metropolitan health services provided staff education on antimicrobial prescribing, compared with very few regional public hospitals and only some private hospitals, with senior medical staff the least likely to receive education. Electronic decision support systems were available in some metropolitan public hospitals, and antibiograms were more commonly available in the private hospital setting.

Perceived barriers to the implementation of AMS programs are outlined in Box 5. Responses were selected from a list of prepared options compiled by the investigators, and respondents could choose multiple options if desired.

\section{Discussion}

With a public hospital response rate of $96.4 \%$, these results may be regarded as census data for the sector, while the private hospital response rate $(67.7 \%)$ can be regarded as representative data. However, with an overall response rate of $84.2 \%$ of eligible Victorian hospitals, this survey provides valuable insight into current AMS activities and highlights key areas for improvement when compared with the ACSQHC AMS strategies.

All health services are required to comply with the NSQHS accreditation standards effective from 1 January 2013, and the role of AMS is clearly outlined in Standard 3.14. ${ }^{6}$ At the time of this survey, hospitals

\section{Characteristics of participating hospitals}

Hospitals, by location

\begin{tabular}{lccc} 
& \multicolumn{3}{c}{ No. (\%) } \\
\cline { 2 - 4 } Location & Public & Private & Total \\
\hline Metropolitan & $32(28.3 \%)$ & $28(66.7 \%)$ & $60(38.7 \%)$ \\
Regional & $81(71.7 \%)$ & $14(33.3 \%)$ & $95(61.3 \%)$ \\
Total & 113 & 42 & 155
\end{tabular}

Public hospitals ( $n=113$ ), by classification"

Classification

No. (\%)

Principal referral

$19(16.8 \%)$

Large major cities

$6(5.3 \%)$

Specialist women's and children's

$2(1.8 \%)$

Large regional and remote

$7(6.2 \%)$

Medium (group 1)

$6(5.3 \%)$

Medium (group 2)

$13(11.5 \%)$

Small regional acute

$22(19.5 \%)$

Small non-acute

$9(8.0 \%)$

Multipurpose services

$9(8.0 \%)$

Unpeered and other 


\section{Number of respondents answering "yes" to the presence of elements of the five essential antimicrobial stewardship strategies, ${ }^{5}$ by hospital type}

No. of respondents (\%)

\begin{tabular}{|c|c|c|c|c|}
\hline Essential strategy & $\begin{array}{l}\text { Public metropolitan } \\
\qquad(n=32)\end{array}$ & $\begin{array}{l}\text { Public regional } \\
\qquad(n=81)\end{array}$ & $\begin{array}{l}\text { Private } \\
(n=42)\end{array}$ & $\begin{array}{c}\text { Total } \\
(n=155)\end{array}$ \\
\hline \multicolumn{5}{|c|}{$\begin{array}{l}\text { Implementing clinical guidelines that are consistent with the latest version of Therapeutic guidelines: antibiotic, }{ }^{13} \text { and which take into account } \\
\text { local microbiology and antimicrobial susceptibility patterns }\end{array}$} \\
\hline $\begin{array}{l}\text { Therapeutic guidelines: antibiotic is available (online or paper-based } \\
\text { copies or both)* }\end{array}$ & $32(100 \%)$ & $81(100 \%)$ & $37(88.1 \%)$ & $150(96.8 \%)$ \\
\hline \multicolumn{5}{|c|}{$\begin{array}{l}\text { Establishing formulary restriction and approval systems that include restricting broad-spectrum and later-generation antimicrobials to patients } \\
\text { in whom their use is clinically justified }\end{array}$} \\
\hline $\begin{array}{l}\text { Antibiotic guidelines are promoted or included in hospital antimicrobial } \\
\text { policy }\end{array}$ & $29(90.6 \%)$ & $37(45.7 \%)$ & $9(21.4 \%)$ & $75(48.4 \%)$ \\
\hline A formulary covering antimicrobials is available & $30(93.8 \%)$ & $31(38.3 \%)$ & $7(16.7 \%)$ & $68(43.9 \%)$ \\
\hline $\begin{array}{l}\text { The formulary specifies restrictions on the use of broad-spectrum } \\
\text { antimicrobials }\end{array}$ & $30(93.8 \%)$ & $14(17.3 \%)$ & $2(4.8 \%)$ & $46(29.7 \%)$ \\
\hline \multicolumn{5}{|c|}{$\begin{array}{l}\text { Reviewing antimicrobial prescribing with intervention and direct feedback to the prescriber - this should, at a minimum, include intensive care } \\
\text { unit patients }\end{array}$} \\
\hline $\begin{array}{l}\text { Feedback is provided to prescriber following the review of antimicrobial } \\
\text { prescription }\end{array}$ & $24(75.0 \%)$ & $41(50.6 \%)$ & $16(38.1 \%)$ & $81(52.3 \%)$ \\
\hline The hospital has a dedicated antimicrobial management team & $7(21.9 \%)$ & 0 & $1(2.4 \%)$ & $8(5.2 \%)$ \\
\hline \multicolumn{5}{|c|}{$\begin{array}{l}\text { Monitoring performance of antimicrobial prescribing by collecting and reporting unit- or ward-specific use data, auditing antimicrobial use and } \\
\text { using quality use of medicines indicators }\end{array}$} \\
\hline Regular audits of antimicrobial prescribing are conducted & $20(62.5 \%)$ & $29(35.8 \%)$ & $22(52.4 \%)$ & $71(45.8 \%)$ \\
\hline $\begin{array}{l}\text { Feedback is provided to prescribers on outcomes of antimicrobial } \\
\text { prescribing audits }\end{array}$ & $13(40.6 \%)$ & $21(25.9 \%)$ & $15(35.7 \%)$ & $49(31.6 \%)$ \\
\hline \multicolumn{5}{|c|}{$\begin{array}{l}\text { Ensuring the clinical microbiology laboratory uses selective reporting of susceptibility testing results that is consistent with hospital } \\
\text { antimicrobial treatment guidelines }\end{array}$} \\
\hline The microbiology service selectively reports antimicrobial sensitivities ${ }^{\dagger}$ & $31(96.9 \%)$ & $74(91.4 \%)$ & $39(92.9 \%)$ & $144(92.9 \%)$ \\
\hline
\end{tabular}

across Victoria are at very different stages of implementing AMS; public metropolitan hospitals are generally well advanced, while there are considerable gaps in public regional and private hospitals.

It is evident that additional work is required in some key areas, such as the implementation and promotion of antimicrobial guidelines through inclusion in hospital policies and staff education programs. There is also a need to establish antimicrobial formularies with restrictions in public regional and private hospitals. These have been shown to improve consumption patterns of broad-spectrum antimicrobials, adverse drug reactions and expenditure, ${ }^{14}$ while reducing the local prevalence of some resistant pathogens. ${ }^{15,16}$ Further, there is an observed gap in the ability to perform postprescription review and audits, including dissemination of findings to prescribing clinicians, an element considered essential for successful AMS. ${ }^{17}$ This shortcoming is possibly due to limited staffing and resources. As lack of training and education in safe and effective antimicrobial use was per- ceived to be the number one barrier to implementing an AMS program in all hospital sectors, there is a need to extend antimicrobial education, particularly to senior clinicians, and to make education an essential strategy rather than supplementary activity.

Lack of resources, including pharmacy, infectious diseases and clinical microbiology services, was among the top three barriers reported. Lack of leadership and unwillingness of doctors to change their prescribing practices were the other major barriers, confirming previous findings. ${ }^{11}$ Building effective workforce capacity requires funding for dedicated AMS staff and activities, and is key to program success and sustainability. Without executive and senior staff support, implementing change into an organisation is challenging. Creating the correct governance structure and developing policies and procedures for the program with support from relevant hospital committees are important change management strategies. ${ }^{18-20}$

These data represent the situation in Victorian health services. However, we expect that other Australian states and territories face similar challenges; there has also been a recent AMS survey of Queensland hospitals with similar findings (Minyon Avent, AMS Pharmacist, Mater Pharmacy Services, Brisbane, personal communication). The introduction of AMS as an accreditation criterion within the new NSQHS standards will play an important role in driving the organisational changes required to meet the challenge of implementing AMS in Australian hospitals. To assist with the gaps and barriers outlined here, the Melbourne Health AMS Research Group is currently undertaking research into developing evidence-based recommendations to guide the national implementation of AMS programs in regional and private hospitals.

\section{Acknowledgements: This study was a collaboration between the Vic DoH and the Melbourne Health AMS Research Group. The AMS Research Group is funded by a National Health and Medical Research Council (NHMRC) project partnership grant; partners include the Victorian Infectious Diseases Service, Vic DoH, Therapeutic Guidelines Ltd and Epworth Richmond. Menino Cotta is supported by an NHMRC postgraduate scholarship. Competing interests: No relevant disclosures. Received 2 Apr 2013, accepted 10 ct 2013. \\ 1 Goossens H. Antibiotic consumption and link to resistance. Clin Microbiol Infect 2009; 15 Suppl 3:} 12-15. 
4 Number of respondents answering "yes" to the presence of elements of the recommended supplementary antimicrobial stewardship strategies ${ }^{5}$ and other governance issues, by hospital type

No. of respondents (\%)

Supplementary strategy

\begin{tabular}{|c|c|c|c|c|}
\hline Supplementary strategy & $\begin{array}{l}\text { Public metropolitan } \\
\qquad(n=32)\end{array}$ & $\begin{array}{l}\text { Public regional } \\
\qquad(n=81)\end{array}$ & $\begin{array}{l}\text { Private } \\
(n=42)\end{array}$ & $\begin{array}{c}\text { Total } \\
(n=155)\end{array}$ \\
\hline Senior medical staff & $12(37.5 \%)$ & $7(8.6 \%)$ & $2(4.8 \%)$ & $21(13.5 \%)$ \\
\hline Junior medical staff & $22(68.8 \%)$ & $9(11.1 \%)$ & 0 & $31(20.0 \%$ \\
\hline Pharmacy & $21(65.6 \%)$ & $6(7.4 \%)$ & $2(4.8 \%)$ & $29(18.7 \%)$ \\
\hline Nursing & $8(25.0 \%)$ & $16(19.8 \%)$ & $9(21.4 \%)$ & $33(21.3 \%)$ \\
\hline No education provided & $5(15.6 \%)$ & $59(72.8 \%)$ & $29(69.0 \%)$ & $93(60.0 \%$ \\
\hline
\end{tabular}

Educating prescribers, pharmacists and nurses about good antimicrobial prescribing practice and antimicrobial resistance

Using information technology such as electronic prescribing with clinical decision support or online approval systems

$\begin{array}{llll}\text { An electronic clinical decision support or approval system is available } \quad 21(65.6 \%) & 0\end{array}$

Annually publishing facility-specific antimicrobial susceptibility data

Antibiograms are provided by the microbiology service $\quad 8(25.0 \%) \quad 8(9.9 \%) \quad 14(33.3 \%) \quad 30(19.4 \%)$

Other governance issues pertaining to good antimicrobial stewardship

There is an antimicrobial prescribing policy in place

$\begin{array}{lcll}29(90.6 \%) & 38(46.9 \%) & 9(21.4 \%) & 76(49.0 \%) \\ 22(68.8 \%) & 11(13.6 \%) & 6(14.3 \%) & 39(25.2 \%)\end{array}$

A dedicated committee to oversee antimicrobial stewardship exists*

Funding is available for antimicrobial stewardship activities

\begin{tabular}{|c|c|c|c|c|}
\hline Pharmacist & 10 (31.3\%) & $1(1.2 \%)$ & 0 & 11 (7.1\%) \\
\hline Medical staff & 9 (28.1\%) & 0 & 0 & $9(5.8 \%)$ \\
\hline
\end{tabular}

* Some regional health services reported that although they did not have a dedicated antimicrobial stewardship committee, other associated committees

such as infection control or drug and therapeutics oversee antimicrobial use at their hospital.

\section{Perceived barriers to antimicrobial stewardship implementation, by hospital type}

\begin{tabular}{|c|c|c|c|c|}
\hline \multirow[b]{2}{*}{ Perceived barrier } & \multicolumn{4}{|c|}{ No. of respondents (\%) } \\
\hline & $\begin{array}{l}\text { Public metropolitan } \\
\qquad(n=32)\end{array}$ & $\begin{array}{l}\text { Public regional } \\
\quad(n=81)\end{array}$ & $\begin{array}{l}\text { Private } \\
(n=42)\end{array}$ & $\begin{array}{c}\text { Total } \\
(n=155)\end{array}$ \\
\hline Lack of training and education in antimicrobial use & $23(71.9 \%)$ & $47(58.0 \%)$ & $26(61.9 \%)$ & $96(61.9 \%)$ \\
\hline Lack of leadership to promote antimicrobial stewardship & $11(34.4 \%)$ & $33(40.7 \%)$ & $19(45.2 \%)$ & $63(40.6 \%)$ \\
\hline Lack of support from senior clinicians & $7(21.9 \%)$ & $12(14.8 \%)$ & $7(16.7 \%)$ & $26(16.8 \%)$ \\
\hline Lack of infectious diseases/clinical microbiology services & $15(46.9 \%)$ & $40(49.4 \%)$ & $18(42.9 \%)$ & $73(47.1 \%)$ \\
\hline Lack of pharmacy resources & $18(56.3 \%)$ & $58(71.6 \%)$ & $14(33.3 \%)$ & $90(58.1 \%)$ \\
\hline Lack of willingness from doctors to change their prescribing practices & $12(37.5 \%)$ & $23(28.4 \%)$ & $18(42.9 \%)$ & $53(34.2 \%)$ \\
\hline Lack of enforcement by hospital management & $12(37.5 \%)$ & $10(12.3 \%)$ & $12(28.6 \%)$ & $34(21.9 \%)$ \\
\hline High level of transient/seconded staff & $11(34.4 \%)$ & $10(12.3 \%)$ & $2(4.8 \%)$ & $23(14.8 \%)$ \\
\hline
\end{tabular}

2 Carlet J, Collignon P, Goldmann D, et al. Society's failure to protect a precious resource: antibiotics. Lancet 2011; 378: 369-371.

3 Högberg LD, Heddini A, Cars O. The global need for effective antibiotics: challenges and recent advances. Trends Pharmacol Sci 2010; 31: 509-515.

4 Owens RC Jr. Antimicrobial stewardship: concepts and strategies in the 21st century. Diagn Microbiol Infect Dis 2008; 61: 110-128.

5 Duguid M, Cruickshank M. Antimicrobial stewardship in Australian hospitals. Canberra: Commonwealth of Australia, 2011. http:// www.safetyandquality.gov.au/wp-content/ uploads/2011/01/Antimicrobial-stewardship-inAustralian-Hospitals-2011.pdf (accessed Oct 2011).

6 Australian Commission on Safety and Quality in Health Care. National Safety and Quality Health Service Standards. Sydney: ACSQHC, 2011. http:// www. safetyandquality.gov.au/wp-content/ uploads/2011/01/NSQHS-Standards-Sept2011.pdf (accessed Oct 2011).

7 Dellit TH, Owens RC, McGowan JE Jr, et al. Infectious Diseases Society of America and the Society for Healthcare Epidemiology of America guidelines for developing an institutional program to enhance antimicrobial stewardship. Clin Infect Dis 2007; 44: 159-177.
8 Tamma PD, Cosgrove SE. Antimicrobial stewardship. Infect Dis Clin North Am 2011; 25 : 245-260.

9 Moody J, Cosgrove SE, Olmsted R, et al. Antimicrobial stewardship: a collaborative partnership between infection preventionists and health care epidemiologists. Am J Infect Control 2012; 40: 94-95.

10 Fishman N. Antimicrobial stewardship. Am JMed 2006; 119 (6 Suppl 1): S53-S61.

11 Chen AWJ, Khumra S, Eaton V, Kong DCM. Snapshot of barriers to and indicators for antimicrobial stewardship in Australian hospitals. JPharm Pract 2011; 41: 37-41.

12 Australian Institute of Health and Welfare. Australian hospital statistics 2010-11. Canberra: AlHW, 2012. (AlHW Cat. No. HSE 117; Health Services Series No. 43.) http://www.aihw.gov.au/ publication-detail/?id=10737421633 (accessed Oct 2013).

13 Antibiotic Expert Group. Therapeutic guidelines: antibiotic. Version 14. Melbourne: Therapeutic Guidelines Limited, 2010.

14 White AC Jr, Atmar RL, Wilson J, et al. Effects of requiring prior authorization for selected antimicrobials: expenditures, susceptibilities, and clinical outcomes. Clin Infect Dis 1997; 25: 230-239.

15 Pakyz AL, Oinonen M, Polk RE. Relationship of carbapenem restriction in 22 university teaching hospitals to carbapenem use and carbapenemresistant Pseudomonas aeruginosa. Antimicrob Agents Chemother 2009; 53: 1983-1986.

16 Pear SM, Williamson TH, Bettin KM, et al. Decrease in nosocomial Clostridium difficile-associated diarrhea by restricting clindamycin use. Ann Intern Med 1994; 120: 272-277.

17 Arnold FW, McDonald LC, Smith RS, et al. Improving antimicrobial use in the hospital setting. Infect Control Hosp Epidemiol 2006; 27: 378-382.

18 Brinsley K, Sinkowitz-Cochran R, Cardo D. An assessment of issues surrounding implementation of the Campaign to Prevent Antimicrobial Resistance in Healthcare Settings. Am J Infect Control 2005; 33: 402-409.

19 Belongia EA, Schwartz B. Strategies for promoting judicious use of antibiotics by doctors and patients. BMJ 1998; 317: 668-671.

20 Leung V, Gill S, Sauve J, et al. Growing a "positive culture" of antimicrobial stewardship in a community hospital. Can J Hosp Pharm 2011; 64: 314-320. 